\title{
Endoscopic submucosal dissection of duodenal adenocarcinoma arising from Brunner's gland
}

\author{
Hidekazu Tanaka, Shigenaga Matsui, Hiroshi Kashida, Masatoshi Kudo
}

Kindai University Faculty of Medicine, Japan

A 70-year-old man was admitted to our hospital because of a duodenal submucosal tumor (SMT) that had been detected during screening gastrointestinal endoscopy. The SMT, which was $10 \mathrm{~mm}$ in diameter with a small orifice at the top of the lesion, was located in the first part of the duodenum. Histopathology of a biopsy from the orifice gave a diagnosis of adenocarcinoma. Therefore, endoscopic submucosal dissection (ESD) was performed to remove the lesion en bloc (Fig. 1). Pathological examination (hematoxylin and eosin staining) of the resected specimen revealed a moderately differentiated tubular adenocarcinoma that had invaded the submucosa, but not the lymphatic vessels or vessels; tumor margins were negative (Fig. 2A,B). Immunohistochemical staining revealed that the carcinoma cells were positive for MUC6 (Fig. 2C), but negative for pepsinogen 1 (Fig. 2D). Thus, this duodenal tumor was confirmed as a duodenal carcinoma arising from Brunner's gland.

Duodenal adenocarcinoma from Brunner's gland is very rare and most cases are treated by surgical resection [1]. Brunner's gland is a mucus-secreting acinar gland located in the submucosa of the duodenum. Therefore, an adenocarcinoma arising from Brunner's gland resembles an SMT. ESD can be

Department of Gastroenterology and Hepatology, Kindai University Faculty of Medicine, Japan

Conflict of Interest: None

Correspondence to: Shigenaga Matsui, MD, PhD, Department of Gastroenterology and Hepatology, Kindai University Faculty of Medicine, Osaka, Japan, e-mail: ma2i@med.kindai.ac.jp

Received 16 January 2019; accepted 23 January 2019; published online 15 February 2019

DOI: https://doi.org/10.20524/aog.2019.0363

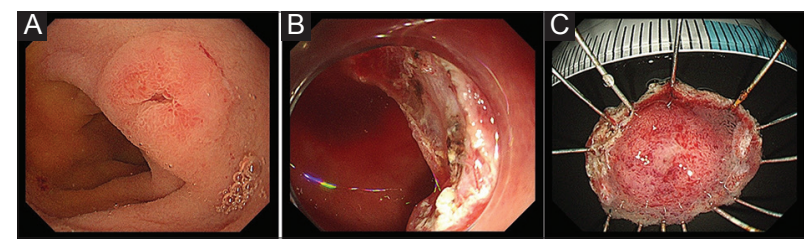

Figure 1 (A) Gastrointestinal endoscopy revealed submucosal tumor with a small orifice in the first part of the duodenum; (B) ulcer after endoscopic submucosal dissection; and (C) specimen

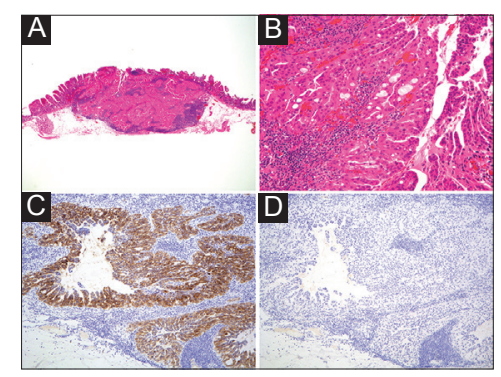

Figure 2 Pathological examination (hematoxylin and eosin staining: (A) $\times 40,(B) \times 400)$ of the resected specimen revealed a moderately differentiated tubular adenocarcinoma that had invaded the submucosa. (C) Immunohistochemical staining revealed that the carcinoma cells were positive for MUC6, but (D) negative for pepsinogen 1

a good treatment selection in adenocarcinoma of duodenum from Brunner's gland.

\section{Reference}

1. Kamei K, Yasuda T, Nakai T, Takeyama Y. A case of adenocarcinoma of the duodenum arising from Brunner's gland. Case Rep Gastroenterol 2013;7:433-437. 